\section{REVISTA}

MEXICANA DE

ECONOMÍA Y

FINANZAS

REMEF

(THE MEXICAN JOURNAL or

ECONOMICS AND FINANCE
Revista Mexicana de Economía y Finanzas, Nueva Época

Volumen 17 Número 2, Abril - Junio 2022, pp. 1-25, e584

DOI: https://doi.org/10.21919/remef.v17i2.584

(Recibido: 22/diciembre/2020, aceptado: 4/abril/2021, publicado: 20/octubre/2021)

\title{
Relaciones de largo plazo entre la política monetaria, el tipo de cambio y el premio al riesgo en México (2003-2018)
}

\author{
Judith Jazmín Castro Pérez ${ }^{1}$ - Universidad Tecnológica de México, México \\ Salvador Cruz Aké - Instituto Politécnico Nacional, México \\ Mario Alejandro Durán Saldívar - Instituto Politécnico Nacional, México
}

El objetivo de esta investigación es estudiar las relaciones de largo plazo entre la política monetaria, el tipo de cambio y el premio al riesgo en la economía mexicana. Mediante la metodología de series de tiempo, modelos ARFIMA y ARFIMAX, con datos diarios de mayo, 2003 a octubre, 2018. Los resultados señalan que las decisiones tomadas por el Banco Central a través de su mecanismo de transmisión (tasa de interés) bajo un objetivo de inflación controlada, envían señales a la economía que impactan en el tipo de cambio, el cual actúa como el canal de transmisión que altera el comportamiento del premio al riesgo de los activos financieros. La recomendación es analizar el impacto que tienen otros mecanismos de política monetaria en el premio al riesgo, la limitación es que sólo se analizaron las relaciones especificas al objetivo, implicando la falta de medición de otros efectos económicos. La originalidad es el análisis de relaciones de largo plazo en la política monetaria mediante modelos fraccionales. En conclusión, se reconoce la existencia de la paradoja del banco central en la economía mexicana.

Clasificación JEL: C32, E5, E52, E58.

Palabras clave: Política Monetaria, Premio al Riesgo, Memoria larga, modelos de series de tiempo.

\section{Long-term Relationship Between Monetary Policy, Exchange Rate and the Risk Premium in Mexico (2003-2018)}

The objective of this research is to study the long-run relationships between monetary policy, the exchange rate, and the risk premium in the Mexican economy. Using the time series methodology, ARFIMA, and ARFIMAX models, with daily data from May 2003 to October 2018. The results suggest that the decisions are taken by the Central Bank through its transmission mechanism (interest rate) under a controlled inflation target, send signals to the economy that impact the exchange rate, acting as the transmission channel that alters the behavior of the risk premium of financial assets. The recommendation is to analyze the impact that other monetary policy mechanisms have on the risk premium, the limitation is that only the relationships specific to the target were analyzed, implying the lack of measurement of other economic effects. The originality is the analysis of long-run relationships in monetary policy using fractional models. In conclusion, the existence of the central bank paradox in the Mexican economy is recognized.

JEL Classification: C32, E5, E52, E58.

Keywords: Monetary Policy, Risk Premium, Long Memory, time series models.

1 Autor de correspondencia. Universidad Tecnológica de México - UNITEC MÉXICO - Campus Querétaro; Email: castro.410210@gmail.com; ORCID: https://orcid.org/0000-0002-3412-2079

* Sin fuente de financiamiento para el desarrollo de la investigación 


\section{Introducción}

En el caso de la economía mexicana las autoridades monetarias tienen como objetivo primordial mantener contralada la inflación y es precisamente bajo este contexto que se envían señales a los individuos que participan dentro del mercado financiero. Si se tiene una postura de política monetaria relajada (baja inflación) los individuos se verán impulsados a incrementar su demanda de riesgo dentro del mercado de valores al prever la situación de la economía agregada como estable, mientras que, por lo contrario si se tiene una postura de política restrictiva (alta inflación) por parte de las autoridades del Banco de México (Banxico), los inversionistas mantendrán bajo cautela sus transacciones en el mercado bursátil, se verán incentivados a reducir su demanda de riesgo pues el entorno que se entrevé presenta mayor inestabilidad.

Es así como se tiene presente la idea de la paradoja del Banco Central, la cual recae en la incongruencia de un estado de aparente estabilidad económica bajo un entorno de inflación controlada por parte del banco central, pero con efectos indeseables en el mercado bursátil, es decir, mayor inestabilidad financiera. Esta paradoja dictamina que la estabilidad tiene como consecuencia la inestabilidad (Minsky, 1977), esto es, gracias a que el sistema en general presenta su punto más riesgoso exactamente en el punto en el que pareciera estar más protegido según la mayoría de los agentes económicos desde su perspectiva individual incentivados por las decisiones y señales de la política monetaria.

Se considera para la economía mexicana de suma importancia reconocer la relación que existe dentro de sus decisiones de política monetaria, con respecto a la tasa de interés, y el entorno financiero en México. Esto con la finalidad de tener una postura que reconozca la forma en la que intervienen las decisiones de las autoridades monetarias en la economía y prever situaciones consideradas como indeseables (mayor riesgo, endeudamiento, crisis, etc.).

Son pocos los estudios empíricos con los que se cuenta para apoyar o refutar la idea anterior al menos en el caso mexicano, la mayor parte de la literatura muestra la evidencia en otras economías. Se piensa en la importancia de realizar el estudio para la economía mexicana con el fin de tener una perspectiva de la relación que existe entre la política monetaria vía tasa de interés y el comportamiento tanto del premio al riesgo de los activos en el mercado de valores mexicano como del tipo de cambio (peso mexicano/dólar americano).

La evidencia que se tiene para la economía norteamericana señala que la presencia de precios crecientes de las acciones, por ejemplo, en julio de 1997, se atribuye a moderadas tasas de interés en el largo plazo y a las expectativas por parte de los inversionistas de que los márgenes de ganancia se mantendrán estables o incluso aumentarán dentro de un ambiente aparentemente seguro y de baja inflación (Greenspan, 1997).

Se sabe que ante la presencia aparente de bajo riesgo se incrementa la demanda de activos, en consecuencia, los precios de los activos dentro del mercado aumentan, el crédito se expande, impulsando los precios hacía arriba; y los inversionistas privados adquieren más riesgos en la búsqueda de rendimiento, todo esto incentivado por la percepción de periodos de bajo riesgo. Por lo que, los periodos de aparentemente bajo riesgo se suelen tener tasas de interés bajas, lo que fomenta la adquisición de mayor riesgo por parte de los inversionistas (Borio, 2011). 
En Bernanke y Gertler (2001) se habla de que los bancos centrales que tienen como objetivo primordial controlar la inflación, se ajustan en automático a las ganancias que provienen de la productividad que eleva los precios de los activos, al mismo tiempo que compensan las disminuciones o los incrementos únicamente especulativos en el valor de las acciones cuyos movimientos a priori se producen mediante la demanda agregada. Su opinión al final es que la estabilidad macroeconómica asociada con la meta de inflación probablemente reducirá el episodio de angustia financiera estimulada por el pánico que podría desestabilizar la economía.

Para el caso de la economía mexicana los estudios sobre la relación entre las decisiones de política monetaria y el valor de los activos financieros aún es escaso. En Salgado, Herrera, y Ramírez (2017) se menciona que el desempeño de la Bolsa Mexicana de Valores, el tipo de cambio y la tasa de interés están ligados de tal forma que cuando ocurre un shock se converge al nivel de equilibrio en mayor tiempo que el sugerido por la cointegración estándar. Esto quiere decir que, aunque las variables mencionadas divergen, existe uno o más puntos en el tiempo en el que convergerán al equilibrio, pero ante la presencia de un shock, los momentos en el tiempo que tardarán para cointegrar serán más que sin la presencia del shock. En otras palabras, el desempeño de la Bolsa Mexicana de Valores, el tipo de cambio y la tasa de interés tardarán más periodos de tiempo en converger cuando existe un shock, que cuando no existe (Salazar-Núñez y Venegas-Martínez, 2015).

En Bordo y Jeanne (2002a) se encontró que la política monetaria óptima depende de las circunstancias económicas en las cuales se incluyen las creencias de los agentes privados. Se dice que la política monetaria óptima actúa en términos generales de tal forma que si se tiene un gran riesgo de quiebra y las autoridades monetarias pueden intervenir desactivándolo a un costo relativamente bajo, se hace referencia a una restricción monetaria proactiva. Se sabe qué a medida que los inversionistas se vuelven más generosos, incrementan los riesgos asociados con una reversión en la confianza del mercado y al mismo tiempo, apoyarse en la ola de optimismo de los inversionistas necesita acciones monetarias más radicales y con un mayor costo. Sin embargo, hoy en día la intención de los responsables de la formulación de políticas no es seguir ese camino, sino perseguir una política reactiva y afrontar las consecuencias de una caída de los precios de las acciones una vez que ocurran (Téllez-León y Venegas-Martínez, 2013).

El objetivo principal de la presente investigación es comprobar si la relación que existe entre las decisiones de política monetaria por parte del Banco de México (vía tasa de interés) y el comportamiento tanto del tipo de cambio como el del premio al riesgo de los activos financieros en México es de largo plazo. En la sección I, se desarrolla la justificación del objetivo de baja inflación por parte de las autoridades monetarias, el uso de la tasa de interés como mecanismo de conducción de la política monetaria y se define al tipo de cambio como el canal de transmisión, además como es que en conjunto las partes mencionadas anteriormente generan efectos en el valor del premio al riesgo de los activos financieros en México. En la sección II, se describen los principales aspectos teórico-metodológicos para trabajar con modelos de integración fraccional, pues este método es considerado el idóneo para obtener las relaciones de largo plazo que se describen dentro del primer capítulo en la presente investigación; las nociones importantes para la investigación son: memoria larga, integración, integración fraccional, modelos ARFIMA y ARFIMAX. En la sección III, se procede a analizar las series económicas de México mediante la metodología descrita en la sección II, con la finalidad de comprobar la existencia de las relaciones de largo plazo entre las variables de estudio y 
validar el impacto de las decisiones de la política monetaria mexicana en el comportamiento del tipo de cambio y el premio al riesgo de los activos financieros mexicanos.

\section{Mecanismo y Canal de Transmisión de la Política Monetaria en México}

La instrumentación de la política monetaria corresponde al conjunto de acciones que lleva a cabo el banco central en los mercados financieros para lograr la estabilidad de precios. Es claro que es muy difícil que el banco central pueda influir en los precios de una economía de manera directa, por lo que para lograrlo éste establece un objetivo operacional ${ }^{2}$ sobre el cual sí se tiene control. Es mediante este objetivo operacional la forma en la que la banca central influye de diversas maneras en la inflación. Para el caso particular del Banco de México, cuando éste establece cierto nivel para la tasa objetivo espera intervenir en el comportamiento de las tasas de interés de largo plazo y afectar así los créditos que otorgan y las tasas que pagan los bancos con el fin de influir en la actividad económica y finalmente tener un impacto en la inflación.

Al respecto Mishkin (1995) menciona que para tener éxito en la conducción de política monetaria, los responsables de esta, deben realizar una evaluación precisa del resultado de sus políticas en la economía. Para ello, es impresindible identificar los mecanismos mediante los cuales la economía es afectada por la política monetaria.

\subsection{La tasa de interés como mecanismo de transmisión de la política monetaria}

Se menciona en que en los últimos cincuenta años la transmisión de la política monetaria a través de mecanismos como el tipo de interés ha sido un detalle en general dentro de la literatura económica. Es así como ha sido considerado por los economistas como la herramienta clave de las autoridades monetarias en la conducción de política económica y también como un pilar del estudio de la macroeconomía (Mishkin, 1995). Así mismo el autor menciona que la visión keynesiana tradicional de cómo un ajuste monetario se transmite en la economía se puede representar como se muestra en la Figura 1,

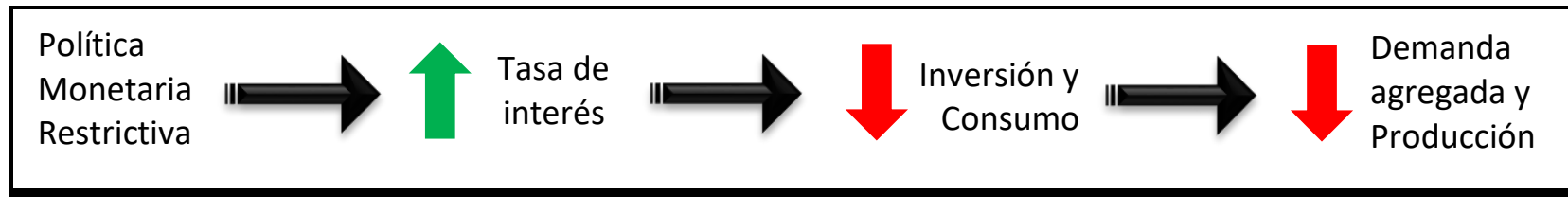

Figura 1. Mecanismo de transmisión de la política monetaria en México Fuente: Creación propia.

2 El objetivo operacional del Banco de México es la tasa de fondeo bancario a plazo de un día. 
Dónde, se indica que una política monetaria restrictiva conduce a un aumento en las tasas reales de interés, que a su vez aumenta el costo del capital, lo que provoca una disminución en el gasto de inversión, y por tanto se convierte en una disminución de la demanda agregada y una caída de la producción.

Por lo que se refiere de manera específica para el caso del Banco de México la herramienta más importante que tiene para controlar el crecimiento de dinero y por lo tanto la inflación, es la tasa de interés. A lo que menciona que el mecanismo funciona de la siguiente manera: "Una mayor tasa de interés reduce la demanda agregada desincentivando la inversión y el consumo, aumentando el ahorro de las personas; de esta manera se limita la cantidad de dinero disponible en la economía, con lo que el nivel de precios disminuye. Lo contrario sucede cuando disminuye la tasa de interés; ahora las personas se ven incentivadas a invertir y consumir, ya que tener el dinero en los bancos no es la mejor opción, por lo que la cantidad disponible en la economía se ve incrementada, lo que hace que el nivel de precios aumente" (Banxico, 2018).

Es así como tomando en cuenta la evolución reciente de la inflación, y también considerando las perspectivas de sus principales determinantes y los pronósticos de esta, cada que se requiere, la Junta de Gobierno decide por unanimidad el objetivo para la tasa de interés interbancaria a un día. Procurando que la postura de política monetaria sea congruente con una tendencia descendente de la inflación anual hacia su meta.

Para Blinder (1998) al tener la política monetaria priorizada por el objetivo de inflación, lo ideal, sería que no se castigue la producción dentro de la actividad económica, pues se piensa que existen alternativas que puedan lograr un desarrollo deseado tanto de la producción como de la inflación, pero para ello se requiere conocer explicitamente cuales son los mecanismos de política monetaria que conducen las decisiones de las autoridades monetarias en la economía, y más aún, de que manera impactan a un nivel agragado de la actividad económica.

\subsection{Canal de transmisión de la política monetaria}

Se entiende como canales de transmisión de la política monetaria aquellas vías por medio de las cuales la tasa de interés de corto plazo puede tener un impacto sobre la oferta y demanda agregada, que le permita posteriormente influir en los precios, y alcanzar así el objetivo de inflación.

Existen diversos factores por los que en la actualidad se ha puesto cada vez mayor atención a los efectos de las decisiones de las autoridades monetarias en el tipo de cambio, los más sobresalientes son dos, primero el gran auge internacional que ha presentado desde hace ya varios años la economía estadounidense, y segundo la llegada del régimen cambiario en el que el valor de la moneda se determina en el mercado sin intervención de los responsables de la política monetaria. Dada la importancia de ambos acontecimientos para la economía se reconoce que existe gran potencial de estudio en el comportamiento del tipo de cambio como subproducto de la trasmisión de la política monetaria.

Para determinar el valor del tipo de cambio de un país es necesario comparar su moneda en relación con la moneda extranjera, pues se busca una equivalencia que permita realizar las transacciones que desean los individuos.

Dada la necesidad de realizar transacciones se reconocen también los efectos que tiene el comportamiento del tipo de cambio en la economía. Si éste se aprecia se encarecerán los activos 
domésticos con respecto a los activos extranjeros, generando una menor demanda de los activos nacionales por parte del sector externo. Pero a su vez, generando que los inversionistas nacionales demanden más de los activos extranjeros pues habrá aumentado el valor de la moneda nacional respecto a la moneda extranjera. Por otro lado, si el tipo de cambio se deprecia, los efectos serán inversos, los activos domésticos serán más atractivos ante las monedas extranjeras, y eso propiciará un aumento en la demanda de estos; mientras que para los inversionistas nacionales representará una disminución al consumo de activos extranjeros.

Ante tal situación se entiende que las fluctuaciones demasiado abruptas pueden no ser deseadas, pues generarían cambios violentos en las transacciones de los inversionistas, y eso se considera como un entorno de inestabilidad en la economía.

Al respecto, para Mishkin (1995) el tipo de cambio también implica efectos de las tasas de interés, porque cuando las tasas de interés reales aumentan, los depósitos en moneda nacional se vuelven más atractivos en comparación con los depósitos en monedas extranjeras, lo que lleva a un aumento en el valor de los depósitos en moneda doméstica, es decir, se tiene una apreciación de la moneda nacional. Ante el aumento del valor de la moneda nacional se tiene que los productos nacionales ahora son más caros que los productos extranjeros, lo que causa una caída de las exportaciones netas y por tanto una baja en la producción total. Lo cual se simplifica en el siguiente esquema (Figura 2),

Política
Monetaria
Restrictiva

Figura 2. Canal de transmisión de la política monetaria

Fuente: Creación propia.

Es necesario especificar que la paridad de poder de compra (PPP) describe la relación entre el tipo de cambio y el nivel de precios de dos economías. Usualmente esta relación se utiliza como un indicador que permite valorar los desajustes teóricos del tipo de cambio. En la presente investigación de asume la existencia de paridad del poder de compra débil para México, lo que permite la existencia de desviaciones de los precios relativos en el tipo de cambio; lo que señala imperfecciones en los flujos de comercio internacional, V.g. el costo del trasporte de las mercancías, los servicios no importables, etc. Esto quiere decir que las fluctuaciones del tipo de cambio tendrían que ser compensadas por las variaciones en los índices de precios de las economías.

De tal forma que esta paridad, señala que si las variaciones de los precios nacionales son mayores a las de los precios extranjeros se dará una apreciación del valor del tipo de cambio. A diferencia, si los cambios de los precios internos son menores a los foráneos, el tipo de cambio se deprecia esto para mantener el poder de compra de la moneda nacional. 


\subsection{La política monetaria y el mercado financiero: Premio al riesgo en México}

Dentro del sistema financiero en los últimos treinta años se han generado múltiples cambios que han modificado la naturaleza de la transacción típica realizada en el mercado accionario, convirtiéndolo en un mercado cada vez más amplio y con una mayor participación que ha permitido considerarlo como un mercado profundo (Rajan, 2006). Así mismo esta extensa participación ha concedido que los riesgos dentro del mercado financiero sean más significativos para la actividad bursátil y a su vez, se tiene un efecto superior en la economía.

Al considerar el riesgo como una situación de incertidumbre, y a su vez la incertidumbre como la falta de certeza sobre algún acontecimiento, se asume que es imposible describir con exactitud más de un resultado posible, un desenlace futuro, o el estado actual. Al respecto Alchian (1950) señala que por definición, "bajo incertidumbre", cada acción que se puede preferir se identifica no unicamente con un resultado, sino con una distribución de posibles resultados. Eso queiere decir que aunque no es posible conocer exactamente el alcance ante la presencia de incertumbre, es posible medir los resultados potenciales por medio de una distribución.

En lo que se refiere a la relación entre política monetaria e incertidumbre en los mercados financieros Bekaert, Hoerova, y Lo Duca (2013) sugiere que el resultado más consistente encuentra que una política monetaria relajada obtendrá aumentos en el apetito de riesgo en periodos futuros, pues se predecirá un entorno de poca incertidumbre en la economía. Dada una decisión de política monetaria relajada los individuos interpretarán un entorno de aparente estabilidad, previendo baja incertidumbre y por ende confiando en una disminución del riesgo en el mercado, y esto desembocara en una mayor demanda de riesgo, propiciando despues de todo incertitumbre. Cabe mencionar que la respuesta inmediata de la incertidumbre a los shocks de política monetaria es más débil que la de la aversión al riesgo, trabajos similares (Gil-León, y Toca- Toca, J. S., 2020), (Vasicek y Venegas-Martínez, 2021).

Hay que mencionar además, restringir la política monetaria ante un boom del mercado de activos puede observarse como un seguro contra el riesgo de una crisis crediticia. Sin embargo, éste seguro es costoso en relación a sacrificios macroeconómicos, pues implica costos inmediatos en términos de una menor producción e inflación. No obstante dejar que el auge se desmarque implica el riesgo de costos aún más grandes en el futuro. Por tanto se entiende que es tarea de las autoridades monetarias valorar los costos y beneficios relativos de una restricción monetaria preventiva ante la presencia de un boom del precio de los activos, así la política monetaria óptima dependerá de estos beneficios y costos. Cabe señalar que con respecto a está idea la visión dominante entre los banqueros centrales se puede identificar como una visión de "negligencia benigna"3(Bordo y Jeanne, 2002b).

En lo que respecta a las burbujas financieras, se tiene una amplia gama de modelos analíticos que sugiere que la política monetaria debe reaccionar ante los movimientos continuos de los precios de las acciones, es decir, se debe reaccionar ante la existencia de una burbuja. Tanto a las burbujas de activos cuya evolución no depende de la política monetaria como a las burbujas cuya probabilidad

\footnotetext{
3 Según éste punto de vista, las autoridades monetarias deberían lidiar con la inestabilidad financiera que podría resultar de un colapso en los precios de los activos siempre y cuando ocurra, pero no deberían ajustar la política monetaria de forma preventiva en la fase de auge (Bordo \& Jeanne, 2002b).
} 
y tamaño si dependan de la política monetaria, es decir, respondiendo más allá de la desviación del crecimiento y el objetivo de inflación, con moderación y cautela (Roubini, 2006).

Dicho de otra forma, las burbujas financieras pueden ser o no motivadas por las decisiones de política monetaria, sin importar cual sea el caso, las autoridades monetarias deben reaccionar de forma cauteloza ante la presencia de alguna burbuja, pues la experiencia menciona que aunque se incurrira en costos para la actividad económica agregada, estos costos serán menores a los que se podría llegar si los responsables de política se limitan a observar el desarrollo de la burbuja.

\subsubsection{Premio al riesgo}

El premio al riesgo se define en la presente investigación como el valor esperado de la rentabilidad de los activos financieros menos el valor esperado de la tasa libre de riesgo (tasa de interés).

La definición del premio al riesgo de éste estudio es tomada de la teoría de portafolios, por lo tanto, se considera que el premio al riesgo de mercado es suficiente para los objetivos de investigación gracias a los siguientes supuestos: a) se cumple la hipótesis de mercado eficiente; b) movilidad perfecta de capitales; c) política monetaria activa; d) no existe arbitraje; e) en el Índice de Sharp, la condición de media - varianza es suficiente para caracterizar el premio al riesgo, y f) la economía analizada tiene un mercado multivariado normal, es decir, cumple con la hipótesis de la paridad de poder de compra en su versión débil.

En otras palabras, el análisis presentado mediante la definición del premio al riesgo corresponde a una medida del comportamiento promedio de la rentabilidad de mercado, dejando de lado los efectos de memoria en los procesos, los cuales crean movimientos, pero no modifican la media (el problema de las martingalas señalado por Mandelbrot).

En este trabajo se propone que, el origen de la volatilidad de los mercados es la necesidad de los inversionistas de generar instrumentos de mayor retorno esperado en ambientes que, en promedio se mantienen estables dada la política monetaria. Esto implica que una política monetaria que excluye la volatilidad de mercado y sus extremos (al estar basada en promedios para los rendimientos de los instrumentos de señalización) genera incentivos para que los agentes económicos creen instrumentos volátiles para compensar la falta de rendimientos por tasas de interés bajas y ambientes, en promedio, estables, de ahí la necesidad de modelar estos retornos con memoria mediante modelos ARFIMA.

Ahora, premio al riesgo se entiende como la tasa de ganancia que los inversionistas adquieren al asumir un riesgo mayor en su inversión, en este caso, lo que los inversionistas consiguen de beneficios por adquirir activos del mercado de capitales y no invertir en los activos libres de riesgo (Engel, 1995).

$$
\mathrm{PR}=\mathrm{r}_{\mathrm{m}}-\mathrm{r}_{\mathrm{l}}
$$

Por otra parte, en la ecuación (1) se tiene la definición teórica del premio al riesgo. Sea PR la tasa de rentabilidad que se adjudica al inversionista por adquirir riesgo de mercado; la tasa de rentabilidad de mercado está definida por $r_{m}$; mientras que $r_{l}$ representa la tasa libre de mercado. 
En otras palabras, el premio al riesgo es el exceso de rentabilidad que adquiere el inversionista al asumir mayor incertidumbre en sus inversiones (Domowitz y Hakkio, 1984).

Según Bomfim (2003) existen dos enfoques distintos en el estudio de la relación entre el mercado de valores y la política monetaria, eston son: los economistas financieros y los economistas monetarios; los financieros durante mucho tiempo han considerado los efectos de las emisiones de datos económicos sobre la volatilidad del mercado de activos a travéz de la observación de lo que ocurre en las fechas de entrada de las noticias con la volatilidad en el mercado de activos; mientras que los monetarios han explorado cómo la política monetaria sorprende al nivel de precios de los activos al relacionar una inesperada decisión de política con el movimiento en el precio de las acciones después del anuncio de la decisión.

\subsection{La paradoja del Banco Central}

Para la economía norteamericana se considera una de las más estables epocas la precedida por la dirección de Alan Greenspan en la Reserva Federal (FED), banco central de los Estados Unidos. Pues para muchos economistas presidió durante casi dos décadas de prosperidad, con solo dos recesiones históricamente breves y suaves en 1990-91 y 2001. Las amenazas importantes que se presentaron para la economía, como la caída del mercado de valores de 1987, la crisis financiera asiática de 199798, la burbuja de las punto.com, el 11/9) fueron moderadas. Se aclamaban las acciones conducidas por las autoridades monetarias como reflejo de la creencia de que se estabilizo la economía sin alimentar nuevamente la inflación, debido a los virtuosos cambios en las tasas de interés y las adecuadas intervenciones en las crisis, todo dirigido por Greenspan (Samuelson, 2013).

Para principios del 2000, se creía en la posibilidad de alcanzar un bienestar más plácido. Los economistas la nombraron "la Gran Moderación”. Greenspan fue su principal arquitecto. Pero había un inconveniente no reconocido: con una economía de bajo riesgo los individuos (propietarios de viviendas, banqueros, administradores de inversiones) conjeturó que podía realizar algo que en una época distinta podría considerarse más arriesgado. Es decir, los consumidores podían obtener más créditos porque el entorno de estabilidad macroeconómica elevaba su capacidad de pagarlos. Las hipotecas para viviendas consideradas "de alto riesgo", las cuales eran concedidas a prestatarios más débiles, se volvieron aparentemente más seguras por considerarse que los precios de las viviendas continuarían subiendo. Los bancos podían asumir más deudas, porque los mercados financieros se encontraban calmados. De ahí, la paradoja Greenspan (o paradoja del banco central): La creencia en un riesgo menor creó más riesgos. El resto es historia (Samuelson, 2013).

También, existe evidencia con respecto a la presencia de precios crecientes de las acciones en julio de 1997, se atribuye a moderadas tasas de interés en el largo plazo y a las expectativas por parte de los inversionistas de que los márgenes de ganancia se mantendrán estables o incluso aumentarán dentro de un entorno aparentemente estable y de baja inflación (Greenspan, 1997). Así, cuando al parecer el riesgo es bajo se incrementa la demanda de activos y por ende los precios de los activos dentro del mercado están aumentando, el crédito se expande impulsando los precios hacía arriba y los inversionistas privados adquieren más riesgos en la búsqueda de rendimiento incentivados por los periodos de percepción de bajo riesgo, además, estos periodos de aparentemente poco riesgo suelen ir acompañados de tasas de interés bajas, fomentando así la adquisición de más riesgos (Borio, 2011). 
En el caso mexicano al respecto de este tema en particular los estudios aún son escasos, sin embargo, si se ha encontrado evidencia en la que las decisiones de política monetaria vía tasa de interés, impacta tanto en el mercado cambiario como en el mercado financiero.

Es congruente pensar que las decisiones del Banco Central que inciden directamente en el comportamiento de la tasa de interés impactan en los niveles del tipo de cambio y posteriormente la volatilidad del mercado cambiario participa en el proceso provocando consecuencias sobre el comportamiento del premio al riesgo de las acciones cotizadas en el mercado financiero en México.

Es decir, sabiendo que en el caso de la economía mexicana las autoridades monetarias tienen como objetivo primordial mantener contralada la inflación, y como mecanismo de transmisión se utiliza la tasa de interés de corto plazo, se piensa que las decisiones de política monetaria envían señales a los individuos que participan dentro del mercado financiero. Siendo así, si se tiene una postura de política monetaria en la que las tasas de interés bajan y con ello se considera una baja en la inflación, los individuos se verán estimulados a incrementar su consumo de riesgo dentro del mercado de valores al prever el entorno de la economía agregada como estable; mientras que, por lo contrario, si se tiene una postura de política restrictiva aumentando la tasa de interés y con ello se tiene la noción de un alza en la inflación, los inversionistas mantendrán bajo cautela sus transacciones en el mercado bursátil, en otras palabras, reducirán su demanda de riesgo pues el entorno que se entrevé presenta mayor inestabilidad.

\section{Metodología que sustenta la paradoja del banco central}

En la presente sección se muestran los principales aspectos teórico-metodológicos para trabajar con modelos de integración fraccional en series de tiempo; explicando nociones importantes para la investigación, como: memoria larga, integración, ruido fraccional gaussiano y cointegración fraccional. Con la finalidad de estimar un ARFIMA (modelo integrado fraccional autorregresivo) que demuestre la existencia de relaciones de largo plazo entre la política monetaria en México y variables consideradas de suma importancia dentro de la economía mexicana.

Específicamente se hace referencia a la tasa de interés a 28 días que funciona como mecanismo de transmisión de las decisiones de la política monetaria en México, y cuyo impacto se da tanto en el tipo de cambio (peso mexicano/dólar americano) como en el premio al riesgo de los activos financieros mexicanos.

\subsection{Memoria larga}

En Baillie (1996) se encuentra que al hallar correlogramas con valores que disminuyen muy lentamente, teniendo como consecuencia que sean significativos durante un gran número de rezagos y por tanto las autocorrelaciones no serán sumables, es posible que se trate de un proceso con memoria larga. Es decir, si tenemos una serie temporal discreta $Y_{t}$ con su correspondiente función de autocorrelación $\rho_{\mathrm{j}}$ en el rezago j, si, 


$$
\lim _{n \rightarrow \infty} \sum_{j=-n}^{n}\left|p_{j}\right|<\infty
$$

Este proceso estocástico (2) conserva una memoria larga. También se puede entender que en la función de densidad espectral $f(\omega)$ del proceso $Y_{t}$ no converge en la frecuencia cero, o, dicho de otra forma, no posee límites a bajas frecuencias.

Cassoni (1994) menciona que en el dominio de las frecuencias en dichos procesos se presenta una función de densidad espectral $f(\lambda)$ positiva, continúa y acotada para $\lambda \in[-\pi, \pi)$. Y además se cumple que:

$$
\sum_{j=0}^{\infty}\left|\gamma_{j}\right|<\infty \text { donde } \gamma_{j}=\operatorname{Cov}\left(p_{t}, p_{t-j}\right)
$$

Esto quiere decir que los rezagos de la serie de tiempo tienen relación entre ellos mismos, pero esta se perderá en el tiempo, no logrando establecer un proceso de caminata aleatoria, pero si identificando uno de memoria larga.

Según Baillie (1996), desde un enfoque empírico basado términos de la persistencia de las autocorrelaciones observadas, la extensión de persistencia es consistente con un proceso esencialmente estacionario, pero en el que las funciones de autocorrelación tardan más en decaer en comparación con los procesos ARMA. Cuando se observa que la función de autocorrelación muestra una persistencia que no es consistente con un proceso I(1) ni un I(0).

\subsection{Integración Fraccional}

La integración fraccional se puede definir a través de entenderla como un proceso $\mathrm{I}(\mathrm{d})$, tal que $\mathrm{d}<$ $1 / 2$ es débilmente estacionario y su función de auto covarianza es no infinita. Siempre que $d>0$, se puede expresar como:

$$
\sum_{j=0}^{\infty}\left|\gamma_{j}\right|<\infty
$$

En esta situación (4) el procedimiento para diferenciar la serie en forma fraccional es desigual al de tomar diferencias enteras ya que ante la sospecha de procesos con memoria larga al diferenciar con números enteros se pierde información de la propia variable y en este caso se considera preciso utilizar todo el pasado de la serie,

$$
(1-L)^{d}=\sum_{j=0}^{\infty} a_{j} L^{j}
$$


donde $a_{j}=\Gamma(j-d) / \Gamma(-d) \Gamma(j+1)$ con $a_{0}=1$ y $\Gamma$ es la función gamma. Tal que para diferenciar la serie fraccionalmente se requiere conocer toda la historia de la serie (teniendo en cuenta que el número de observaciones $\mathrm{N}$ es siempre finito), entonces la serie diferenciada en forma fraccional se obtiene mediante,

$$
I F_{t}=\sum_{j=0}^{t-j} a_{j} I_{t-j}
$$

Al obtener un proceso integrado de forma fraccional estacionario e invertible los valores del orden de diferenciación d deben de estar entre $-1 / 2$ y $1 / 2$. Si d es mayor que $1 / 2$ el proceso es no estacionario y si es menor que $-1 / 2$ el proceso es no invertible (Véase en la Cuadro 1 ).

Cuadro 1. Procesos de integración fraccional

\begin{tabular}{|c|l|}
\hline $\begin{array}{c}\text { Orden de } \\
\text { integración }\end{array}$ & \multicolumn{1}{|c|}{ Modelo de caso. } \\
\hline$-1 / 2<\mathrm{d}<0$ & $\begin{array}{l}\text { Se tiene un proceso con memoria negativa (el proceso es } \\
\text { antipersistente). }\end{array}$ \\
\hline $0<\mathrm{d}<1 / 2$ & Se tiene un proceso con memoria larga (el proceso es persistente). \\
\hline $\mathrm{d}=0$ & Se tiene un proceso con memoria corta. \\
\hline
\end{tabular}

Fuente: Creación propia con información obtenida en Baillie (1996).

Dentro de la presente investigación dado que se plantea como objetivo establecer las relaciones de largo plazo entre las variables de estudio, es de suma importancia identificar que las series a estudiar dependen fuertemente de su historia, es decir, poseen memoria larga, y además bajo la sospecha de que su orden de integración es fraccional.

\subsection{Modelos ARFIMA}

En la presente sección se desarrolla la especificación del modelo ARFIMA desde su forma funcional y su aplicabilidad en esta investigación. Este método se estima en dos fases, la primera partiendo de su forma simple (ARFIMA) y la segunda asumiendo variables exógenas (ARFIMAX).

El modelo "Autorregresivo fraccionalmente integrado" (ARFIMA), en el cual se desarrolla el estudio de una serie de tiempo que tiene la condición de memoria larga, la cual de acuerdo con la metodología empleada es una variable con un orden de integración fraccional; en otras palabras, de la serie estimada que depende fuertemente del pasado se busca entender su comportamiento con los componentes autorregresivo y media móvil, reconociendo el concepto de diferenciación fraccional, desarrollado en Adenstedt (1974), Hosking (1981) y Granjera (1980).

En Blanco (2006) se tiene la representación del proceso ARMA (para el tipo de situación mencionada anteriormente) de la siguiente forma: 


$$
\varphi(\mathrm{L})(1-\mathrm{L})^{\delta} \mathrm{y}_{\mathrm{t}}=\theta(\mathrm{L}) \mathrm{e}_{\mathrm{t}}
$$

En (7), $\varphi(\mathrm{L})$ representa la parte autorregresiva o la dependencia de los valores actuales a los valores del pasado. Por otra parte, $\theta(\mathrm{L})$ son los errores de pronóstico o innovaciones de la metodología. Y ambas representan el polinomio de rezagos de cada proceso, esto es importante porque al obtener las raíces de ambos procesos estas se encuentran fuera del círculo unitario, lo que permite que sea estacionario e invertible.

En el caso de $(1-\mathrm{L})^{\delta}$ representa el operador de rezagos o de diferenciación, tal que, para que $Y_{t}$ sea estacionario, el coeficiente de diferenciación fraccional debe ser menor a un medio $(\delta<1 / 2)$ y para que sea invertible ser mayor a menos un medio $(-1 / 2<\delta)$.

Por lo tanto, el intervalo debe ser $\delta \epsilon(-1 / 2,1 / 2)$ para que $Y_{t}$ cumpla con sus criterios base. En el caso de que $1 / 2 \leq \delta<1$ entonces existe no estacionariedad de $\mathrm{Y}_{\mathrm{t}}$; esto indica que, aunque seguirá una dinámica que converge a su media, el proceso no es de memoria larga.

Para el caso en el que $\delta \geq 1$ la serie es no es estacionaria y por lo tanto no retorna a su comportamiento promedio entrando en el problema de la divergencia. Y para $\delta=0$, se tiene que el proceso es una estructura ARMA, en otras palabras $Y_{t}$ es una serie de tiempo estacionaria con memoria corta.

Reconociendo lo anterior, el proceso bajo el cual se dio respuesta a la hipótesis del presente trabajo es un modelo ARFIMA en su forma simple y también con entradas exógenas (ARFIMAX). Para explicar las relaciones de largo plazo entre la política monetaria en México y variables económicas consideradas de suma importancia como lo son el tipo de cambio (peso mexicano/dólar americano) y el premio al riesgo de los activos financieros mexicanos, se debe tener en cuenta todas las relaciones inmersas en el panorama de estudio, es decir, tanto de forma individual como conjunta.

El modelo ARFIMAX presenta aportaciones interesantes dentro de la investigación, por ejemplo, medir las implicaciones conjuntas del pasado de las variables de interés (consideradas integradas fraccionalmente) con la historia de alguna otra variable (incluso que no necesariamente presente un orden de integración fraccional). Permitiendo una visión aún más completa de la dinámica entre variables significativas dentro de la economía mexicana.

Por lo tanto, el modelo ARFIMAX está especificado en la siguiente ecuación (Sriboonchitta et al., 2011).

$$
\mathrm{y}_{\mathrm{t}}=\alpha_{0}+\alpha_{1} \mathrm{y}_{\mathrm{t}-1}+\cdots+\alpha_{\mathrm{p}} \mathrm{y}_{\mathrm{t}-\mathrm{p}}+\beta_{1} \mathrm{x}_{\mathrm{t}}+\cdots+\beta_{\mathrm{d}} \mathrm{x}_{\mathrm{t}-\mathrm{d}}+\varepsilon_{\mathrm{t}}-\theta_{1} \varepsilon_{\mathrm{t}-1}-\cdots-\theta_{\mathrm{q}} \mathrm{y}_{\mathrm{t}-\mathrm{q}}
$$

En (8), $\left(\alpha_{1} \mathrm{y}_{\mathrm{t}-1}+\cdots+\alpha_{\mathrm{p}} \mathrm{y}_{\mathrm{t}-\mathrm{p}}\right)$ es la parte autorregresiva de la serie estimada y $\left(\beta_{1} \mathrm{x}_{\mathrm{t}}+\cdots+\right.$ $\left.\beta_{\mathrm{d}} \mathrm{x}_{\mathrm{t}-\mathrm{d}}\right)$ muestra la parte exógena del modelo, por último, $\left(-\theta_{1} \varepsilon_{\mathrm{t}-1}-\cdots-\theta_{\mathrm{q}} \mathrm{y}_{\mathrm{t}-\mathrm{q}}\right)$ representa las innovaciones del proceso (Titus, Wanjoya, y Mageto, 2021) y (Izzeldin, Hassan, Pappas, y Tsionas, 2019). 


\section{Paradoja del banco central caso México}

La herramienta por excelencia que funge como mecanismo de transmisión de la política monetaria en México es la "Tasa de interés a 28 días" en el estudio se utilizan el rendimiento de CETES a 28 días, y es considerada para la presente investigación como la variable independiente pues su valor es establecido por decisión de las autoridades monetarias bajo el objetivo de inflación controlada.

En la presente investigación se asume que, ante la presencia de un entorno aparentemente estable en la economía mexicana, propiciado por las decisiones de política monetaria, los agentes económicos se ven incentivados a consumir una mayor cantidad de riesgo dentro del mercado financiero y de igual forma dentro del mercado cambiario se tienen repercusiones bajo los movimientos de la tasa de interés. Se realiza un estudio de la variable "Tasa de interés a 28 días" con información diaria, los datos se obtuvieron de la página oficial del banco de datos de Banxico, y el periodo de estudio es de mayo del 2003 a octubre del 2018 (Gráfica 1).

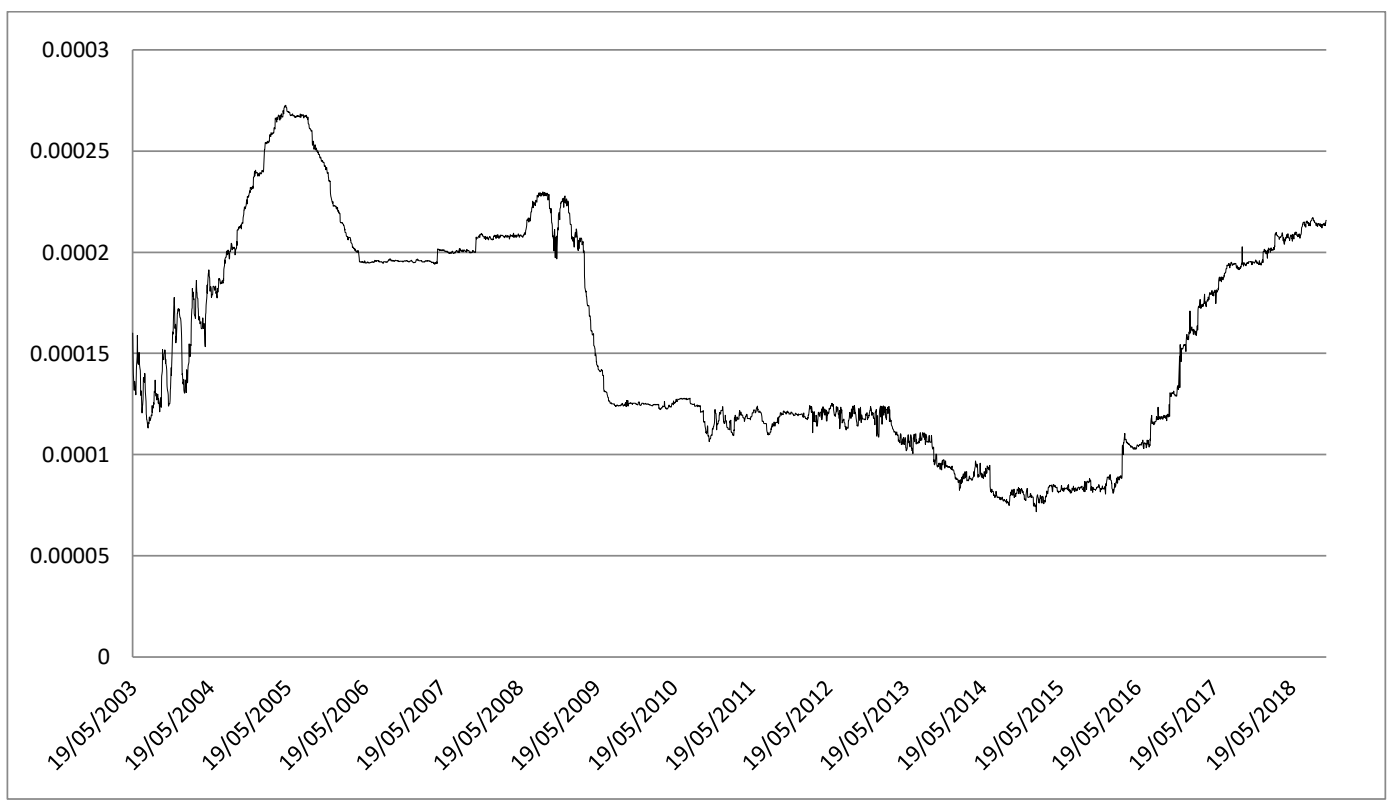

Gráfica 1. Tasa de interés a 28 días, considerada como el reflejo de las decisiones de política monetaria por parte del Banco de México

Fuente: Creación propia con datos de la página oficial del banco de datos de Banxico.

El canal de transmisión más común de la política monetaria en México y el que importa para fines de esta investigación es el “Tipo de cambio (peso mexicano/dólar americano)". Ante los movimientos de la tasa de interés en México, los participantes en el mercado extranjero toman decisiones que terminan repercutiendo dentro de la estabilidad de la economía mexicana. 


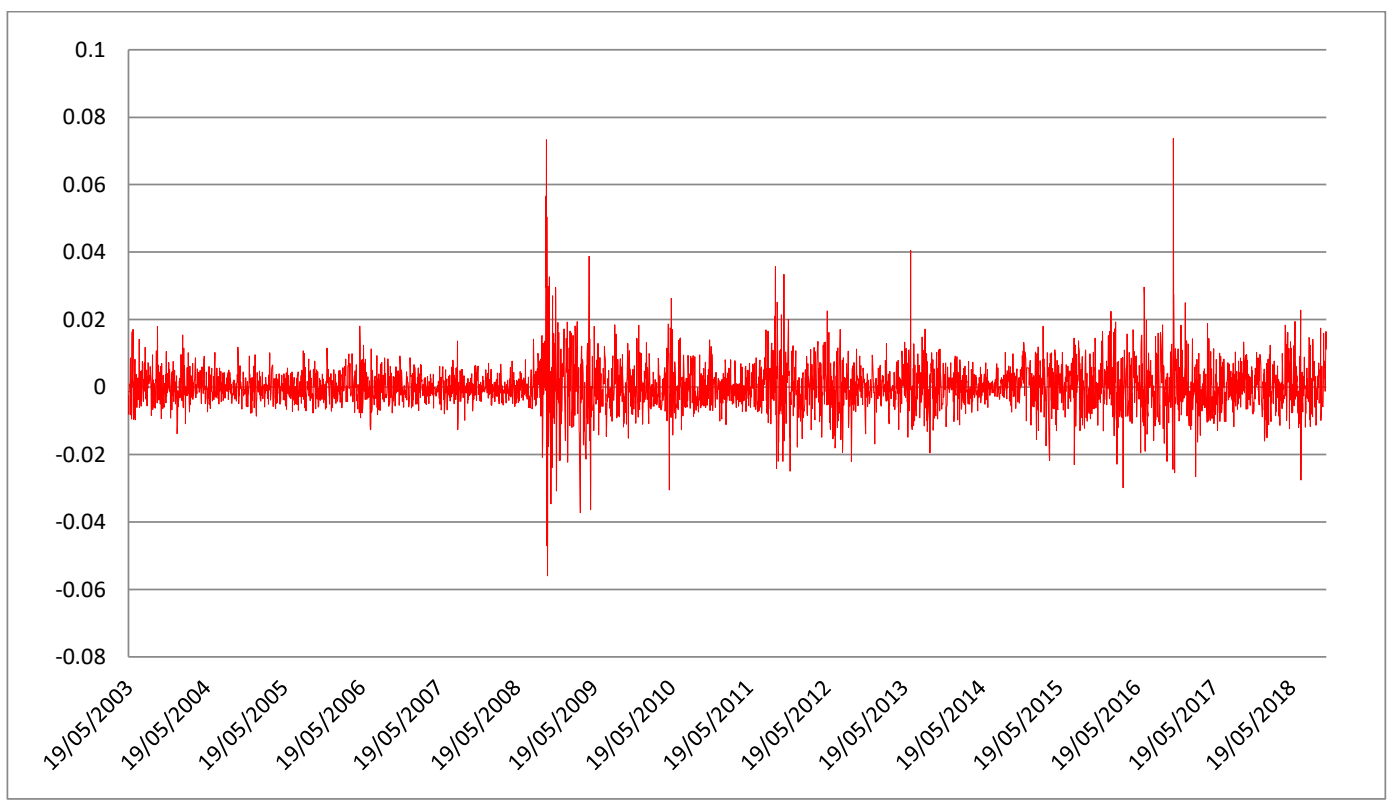

Gráfica 2. Tipo de cambio (peso mexicano/dólar americano) considerado como canal de transmisión de las decisiones de política monetaria en México

Fuente: Creación propia con datos de la página oficial del banco de datos de Banxico.

Se realiza un estudio de la variable "Tipo de cambio (peso mexicano/dólar americano)" con información diaria, los datos se obtuvieron de la página oficial del banco de datos de Banxico, y el periodo de estudio es de mayo del 2003 a octubre del 2018 (Gráfica 2).

El mercado financiero en México es un mercado poco profundo, aún en auge, pero que sin duda es capaz de reflejar la perspectiva de los individuos de la actividad económica en el país. Tiene un indicador mediante el cual le es posible otorgar valores para determinar día con día si el desempeño de la Bolsa Mexicana de Valores tuvo una evolución o un retroceso.

Se realiza el estudio de la variable "Premio al riesgo" que se calculó mediante la diferencia entre el rendimiento del Índice de precios y cotizaciones de la Bolsa Mexicana de Valores (S\&P/ BMV IPC) considerado como la rentabilidad de los activos financieros con riesgo menos la rentabilidad de los activos libres de riesgo. Información diaria, los datos se obtuvieron de la página Yahoo! Finance, y el periodo de estudio es de mayo del 2003 a octubre del 2018 (Gráfica 3). 


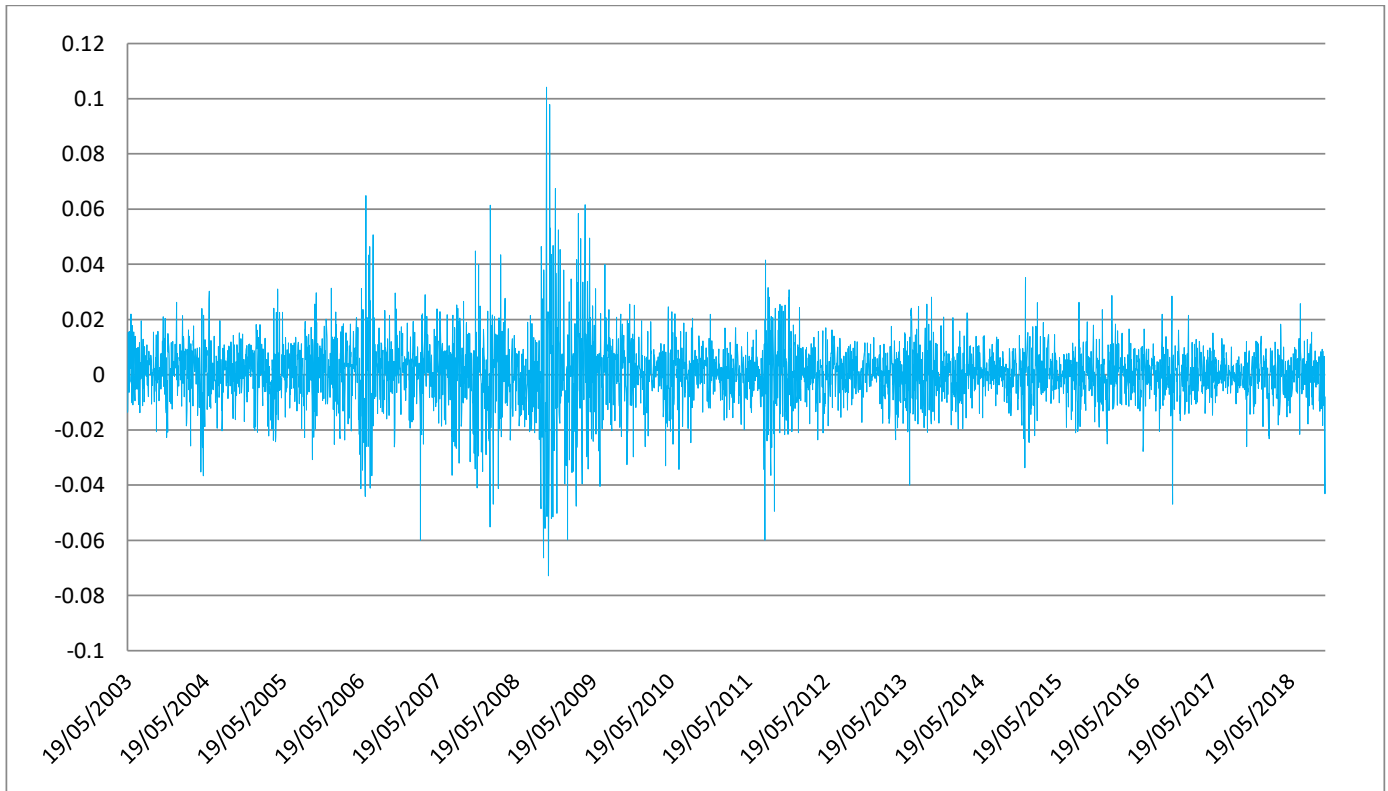

Gráfica 3. Premio al riesgo dentro del mercado financiero en la economía mexicana Fuente: Creación propia con datos de la página Yahoo Finance.

De acuerdo con esta investigación, la cual se centra en identificar las relaciones de largo plazo entre la política monetaria en México, el tipo de cambio (peso mexicano/ dólar americano) y el premio al riesgo de los activos financieros mexicanos, se considera importante observar su comportamiento en conjunto (Gráfica 4). Este comportamiento demuestra que existen momentos en los que, tiempos de gran volatilidad son precedidos por movimientos en la tasa de interés.

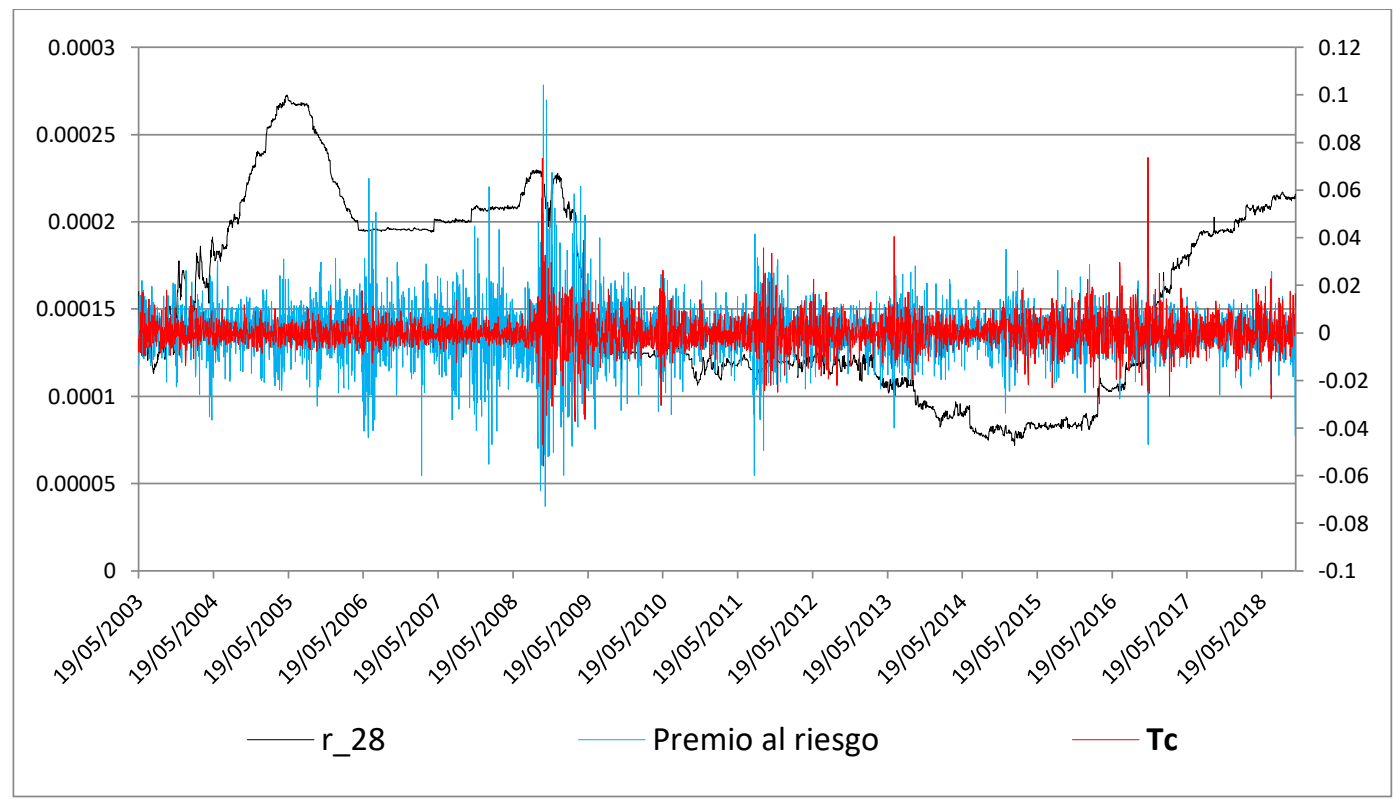

Gráfica 4. Premio al riesgo, Tipo de cambio y Tasa de interés en la economía mexicana Fuente: Creación propia con datos de la página Yahoo Finance. 


\subsection{Resultados Modelos ARFIMA.}

En este apartado se desarrolla la aplicación de los modelos ARFIMA con el objetivo de reconocer la existencia de memoria larga en las variables de estudio. El Cuadro 2 muestra el modelo ARFIMA de la tasa de interés, y señala que la memoria larga es parte fundamental para entender el comportamiento de esta variable, en otras palabras, que las decisiones que toma el Banco de México reflejadas a través de esta variable consideran la información de movimientos anteriores en la política monetaria. Entonces, dado que el coeficiente fraccional " $D$ " es significativo, se sugiere que dada la presencia de memoria larga este modelo permite explicar de una mejor forma los valores presentes de la serie.

Cuadro 2. Modelo ARFIMA de la tasa de Interés

\begin{tabular}{|l|c|}
\hline \multicolumn{2}{|c|}{ Tasa de interés } \\
\hline Variable & Coeficiente \\
\hline D & $0.07302415^{* * *}$ \\
\hline AR $(1)$ & $-0.68070971^{* * *}$ \\
\hline AR $(2)$ & $-0.13562044^{* * *}$ \\
\hline MA (1) & $0.54042768^{* * *}$ \\
\hline $\begin{array}{l}\text { Estadísticamente significativo al } 99 \%\left(^{* * *}\right), 95 \% \\
\left({ }^{* *}\right) \text { y } 90 \%\left({ }^{*}\right) .\end{array}$ \\
\hline
\end{tabular}

Fuente: Elaboración propia en el software estadístico econometric-views.

En el Cuadro 3 se muestra el modelo ARFIMA del premio al riesgo en el que se encontró que el incentivo al riesgo de los inversionistas tiene relación con su pasado inmediato, esto por el poder explicativo que presenta su componente autoregresivo; pero también depende en cierto grado de su historia, esto sugerido por que el valor del coeficiente de integración fraccional resulta ser estadísticamente significativo. Además, el componente de media móvil sugiere que existe información exógena al comportamiento de la variable que impacta de forma inversamente proporcional al valor presente del premio al riesgo.

Cuadro 3. Modelo ARFIMA del premio al riego
\begin{tabular}{|l|c|}
\hline \multicolumn{2}{|c|}{ Premio al riesgo } \\
\hline Variable & Coeficiente \\
\hline D & $0.09758718^{* *}$ \\
\hline AR (1) & $0.77294892^{* * *}$ \\
\hline AR (2) & $-0.07889649^{* * *}$ \\
\hline MA (1) & $-0.78839316^{* * *}$ \\
\hline $\begin{array}{l}\text { Estadísticamente significativo al } 99 \%\left(^{* *}\right), 95 \% \\
(* *) \text { y } 90 \%\left(^{*}\right) .\end{array}$ \\
\hline
\end{tabular}

Fuente: Elaboración propia en el software estadístico econometric-views.

El Cuadro 4 muestra el modelo ARFIMA del tipo de cambio. Este modelo señala la presencia de memoria larga en la variable tipo de cambio, indicada por el coeficiente de integración fraccional 
estadísticamente significativo. A su vez, se observa que tanto el componente autorregresivo como el de media móvil tienen poder explicativo dentro la variable, lo que es de esperarse dado que se tuvo bajo sospecha desde un principio que los valores presentes del tipo de cambio obedecen tanto al cambio de factores exógenos como al generado dentro de su propia historia.

Cuadro 4. Modelo ARFIMA del Tipo de cambio

\begin{tabular}{|l|c|}
\hline \multicolumn{2}{|c|}{ Tipo de cambio } \\
\hline Variable & Coeficiente \\
\hline D & $-0.03321325^{* * *}$ \\
\hline AR (1) & $-0.76947011^{* * *}$ \\
\hline AR (2) & $0.10723015^{* * *}$ \\
\hline MA (1) & $0.86389882^{* * *}$ \\
\hline $\begin{array}{l}\text { Estadísticamente significativo al } 99 \%\left(^{* *}\right), 95 \% \\
(* *) \text { y } 90 \%\left(^{*}\right) .\end{array}$ \\
\hline
\end{tabular}

Fuente: Elaboración propia en el software estadístico econometric-views.

\subsection{Resultados Modelos ARFIMAX}

La presente sección desarrolla los modelos ARFIMAX, estos son modelos de memoria larga que permiten identificar aquellos componentes existentes en la historia de la serie de tiempo que afectan su comportamiento actual, además en este caso se agregan otras variables que permitan identificar causalidad con la serie analizada. Los modelos desarrollados en la presente sección indican la dependencia de las series estudiadas a la historia de estas, esto porque los coeficientes de integración fraccional denotados por D son estadísticamente significativos en todos los modelos realizados.

El Cuadro 5, muestra el modelo ARFIMAX del premio al riesgo en función de la tasa de interés y el tipo de cambio. La estimación de las variables en conjunto presenta la relación causal entre el mecanismo de transmisión y el canal de transmisión de la política monetaria, contra el exceso de rendimiento que proporciona el mercado. Se encontró que la tasa de interés (mecanismo) es estadísticamente no significativa, es decir, las decisiones de política monetaria no tienen una relación directa.

Por otra parte, la volatilidad del tipo de cambio es significativa en su valor actual y su primer rezago tomando coeficientes con signo negativo. En otras palabras, los movimientos en la tasa de interés pierden efecto en premio al riesgo, debido a que su efecto es incorporado en el canal de transmisión en el periodo corriente y en un rezago. Esto implica que, en promedio, los agentes económicos incorporan los movimientos de la política monetaria, aunque los movimientos abruptos y las agrupaciones de volatilidad (memoria larga) persisten, de hecho, son usados para generar ingresos dentro del mercado.

Lo anterior indica que la tasa de interés en un modelo conjunto pierde poder explicativo sobre el valor del premio al riesgo de los activos financieros mexicanos, lo que es un tanto esperado dado que se piensa que la información proporcionada a través del tipo de cambio como canal de transmisión es suficiente para dar respuesta al comportamiento de la serie, incluso se observa como 
la rentabilidad del tipo de cambio impacta de manera inversamente proporcional en el premio al riesgo, lo que es congruente con la literatura estudiada, pues ante una disminución de la tasa de interés e inflación baja, los individuos estiman la economía agregada como estable y segura, eso desencadena en un incremento del consumo de riesgo dentro del mercado de valores y a su vez un aumento del valor del premio al riesgo; en caso de un aumento en la tasa de interés los resultados serán completamente contrarios.

Cuadro 5. Modelo ARFIMAX 1

\begin{tabular}{|l|c|}
\hline \multicolumn{2}{|c|}{$\begin{array}{c}\text { Premio al Riesgo en función de la tasa de } \\
\text { interés y el tipo de cambio }\end{array}$} \\
\hline Variable & Coeficiente \\
\hline D & $-0.038385^{* * *}$ \\
\hline DR & -39.64363 \\
\hline TC & $-0.637988^{* * *}$ \\
\hline TC $(-1)$ & $-0.072212^{* * *}$ \\
\hline AR $(1)$ & $0.052765^{* * *}$ \\
\hline $\begin{array}{l}\text { Estadísticamente significativo al } 99 \%\left(^{* * *}\right), 95 \% \\
(* *) \text { y } 90 \%(*) .\end{array}$ \\
\hline
\end{tabular}

Fuente: Elaboración propia en el software estadístico econometric-views.

En el Cuadro 6 se muestra la estimación del modelo ARFIMAX del premio al riesgo en función de la tasa de interés. La primera consideración es que el modelo muestra la existencia de memoria larga en la forma funcional planteada y entonces es necesario modelar el comportamiento de la serie de tiempo a partir de integración fraccional. Además, a diferencia del modelo conjunto (Cuadro 5) en este caso se encontró que la tasa de interés si tiene poder explicativo a un nivel de confianza del 10\%.

En este caso, la tasa de interés es inversamente proporcional al premio al riesgo, lo que indica que si se tiene una postura de política monetaria relajada (disminución de la tasa de interés), los individuos incrementan su demanda de riesgo dentro del mercado de valores al prever la situación de la economía agregada como estable, aumentando así el valor del incentivo al riesgo de participar en el mercado financiero; mientras que, por lo contrario, si se tiene una postura de política restrictiva por parte de las autoridades del Banco de México (alza de la tasa de interés), los inversionistas reducirán su demanda de riesgo pues el entorno que se prevé presenta inestabilidad, cuya consecuencia será la disminución del valor del premio al riesgo financiero en la economía mexicana.

Cuadro 6. Modelo ARFIMAX 2

\begin{tabular}{|l|c|}
\hline \multicolumn{2}{|c|}{ Premio al Riesgo en función de la tasa de } \\
Interés
\end{tabular}




\begin{tabular}{|l|l|}
\hline MA (1) & $-0.79007054^{* * *}$ \\
\hline Estadísticamente significativo al 99\% (***), 95\% \\
$\left(^{* *}\right)$ y $90 \%(*)$.
\end{tabular}

Fuente: Elaboración propia en el software estadístico econometric-views.

La prueba impulso respuesta del premio al riego en función de la tasa de interés (Figura 3) sugiere que ante una fluctuación en la tasa de interés el premio al riesgo es afectado de forma inmediata pero este efecto es transitorio, dado que la variable regresa a su trayectoria. Esto muestra que la tasa de interés tiene un efecto inmediato o en otras palabras que en efecto se trata de un mecanismo de transmisión y no un canal.
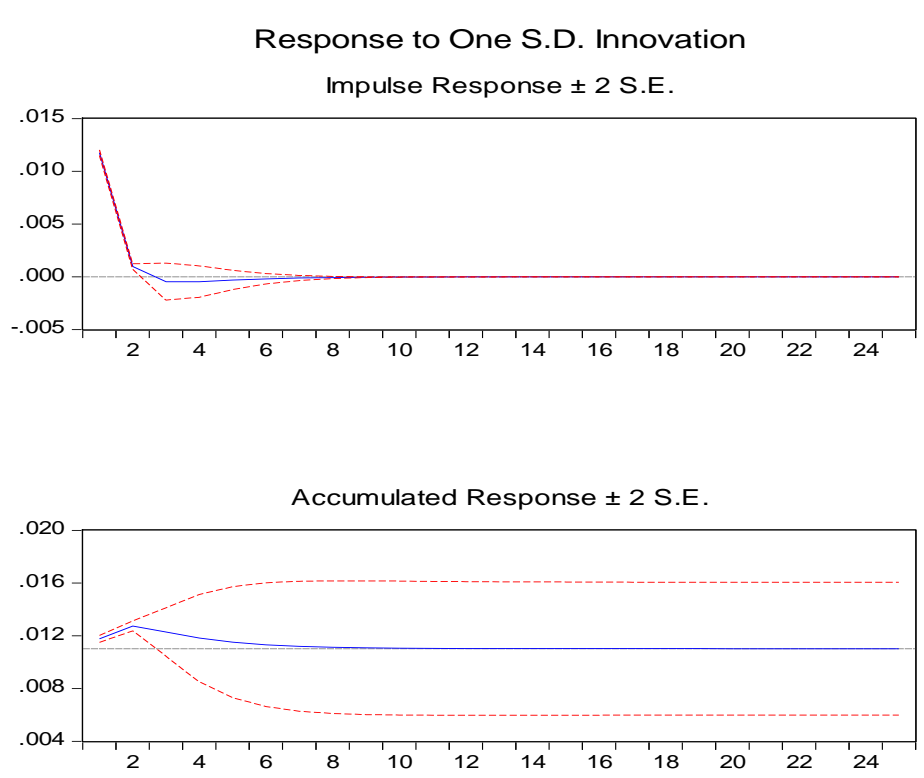

Figura 3. Prueba impulso respuesta del premio al riego en función de la tasa de interés Fuente: Elaboración propia en el software estadístico econometric-views.

El Cuadro 7 muestra el modelo ARFIMAX del premio al riesgo en función del tipo de cambio. Se encontró que la rentabilidad del tipo de cambio es inversamente proporcional al incentivo al riesgo de los inversionistas. Esto se explica porque ante mayor rentabilidad en el mercado cambiario propiciada por una política monetaria restrictiva, los inversionistas prevén un entorno inestable dados los aumentos de la tasa de interés y se ven desalentados a adquirir activos con mayor riesgo por lo que al disminuir la participación dentro del mercado financiero se tiene una disminución del premio al riesgo, mientras que por lo contrario ante una disminución de la rentabilidad del tipo de cambio se conseguirán efectos inversos, situación que refiere a un incremento del premio al riesgo de los activos financieros mexicanos. 
Cuadro 7. Modelo ARFIMAX 3

\begin{tabular}{|l|r|}
\hline \multicolumn{2}{|c|}{ Premio al Riesgo en función del tipo de } \\
cambio
\end{tabular}

Fuente: Elaboración propia en el software estadístico econometric-views.

La prueba impulso respuesta del premio al riego en función del tipo de cambio (Figura 4) señala que ante una fluctuación en el tipo de cambio existe un efecto de tipo permanente en el premio al riesgo, tal como se observa en la gráfica de respuesta acumulada, situación que se preveía pues se anticipaba que es el tipo de cambio el que funge como el canal de transmisión de la política monetaria.
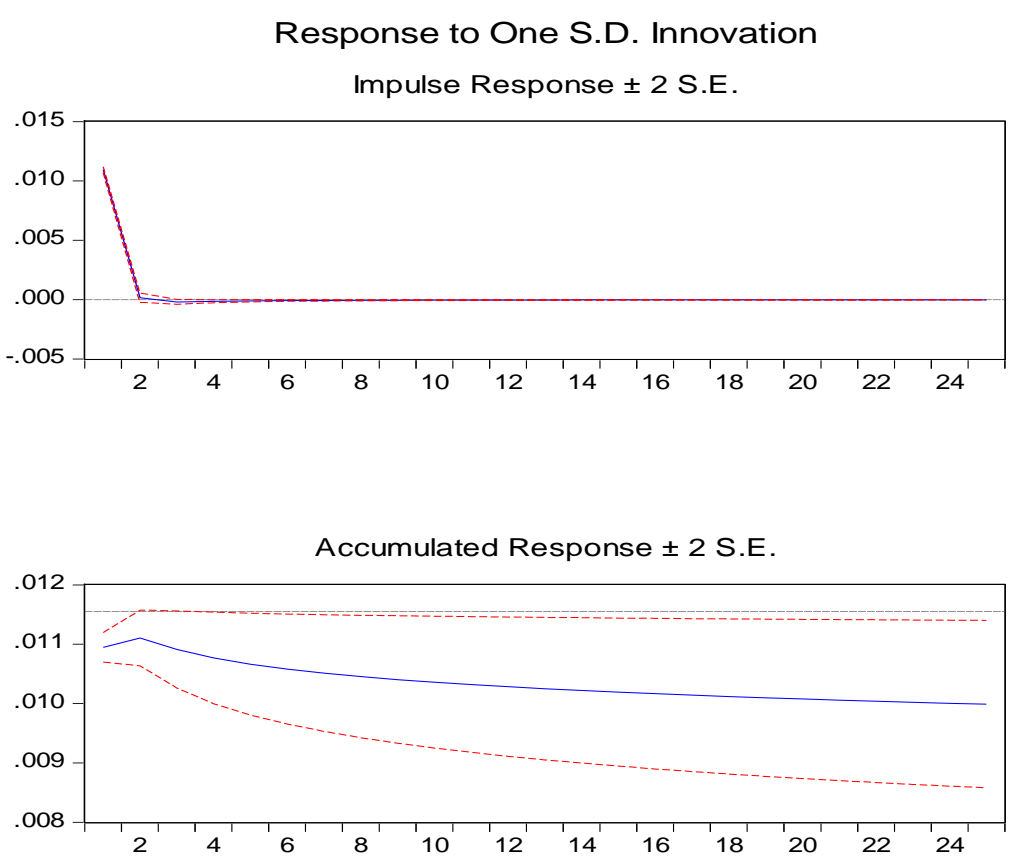

Figura 4. Prueba impulso respuesta del premio al riego en función del tipo de cambio Fuente: Elaboración propia en el software estadístico econometric-views.

Continuando con el análisis, en la Cuadro 8 se tiene el modelo ARFIMAX del tipo de cambio en función de la tasa de interés, en el cual la tasa de interés resulta ser estadísticamente significativa y afecta de forma directa la volatilidad del tipo de cambio, además muestra que éste impacto es grande dado que el coeficiente es de 204.47. Lo que indica que ante movimientos de la política monetaria a través de su mecanismo de transmisión se genera un efecto importante y además directamente proporcional en la rentabilidad del tipo de cambio. Por ejemplo, ante una decisión de 
política monetaria relajada por parte del Banco Central (disminución de la tasa de interés) los depósitos en moneda extranjera se vuelven más atractivos en comparación con los de moneda nacional, lo que lleva a un aumento en el valor de los depósitos en moneda extranjera y una disminución en el valor de los depósitos en moneda doméstica, por tanto se tiene una depreciación de la moneda nacional, es decir menor rentabilidad del tipo de cambio.

Lo anterior es congruente con el análisis planteado en la investigación dado que se justifica que la tasa de interés como mecanismo de transmisión de las decisiones del banco central este fuertemente relacionada con el canal de transmisión de la política monetaria, que en este caso se trata de la rentabilidad del mercado cambiario.

Cuadro 8. Modelo ARFIMAX 4

\begin{tabular}{|l|r|}
\hline \multicolumn{2}{|c|}{ Tipo de cambio en función de la tasa de } \\
interés
\end{tabular}

Fuente: Elaboración propia en el software estadístico econometric-views.

La prueba impulso respuesta del tipo de cambio en función de la tasa de interés (Figura 5) muestra que, ante una innovación en la tasa de interés, existe un efecto de tipo permanente en la volatilidad del tipo de cambio, tal como de observa en la gráfica de respuesta acumulada. 

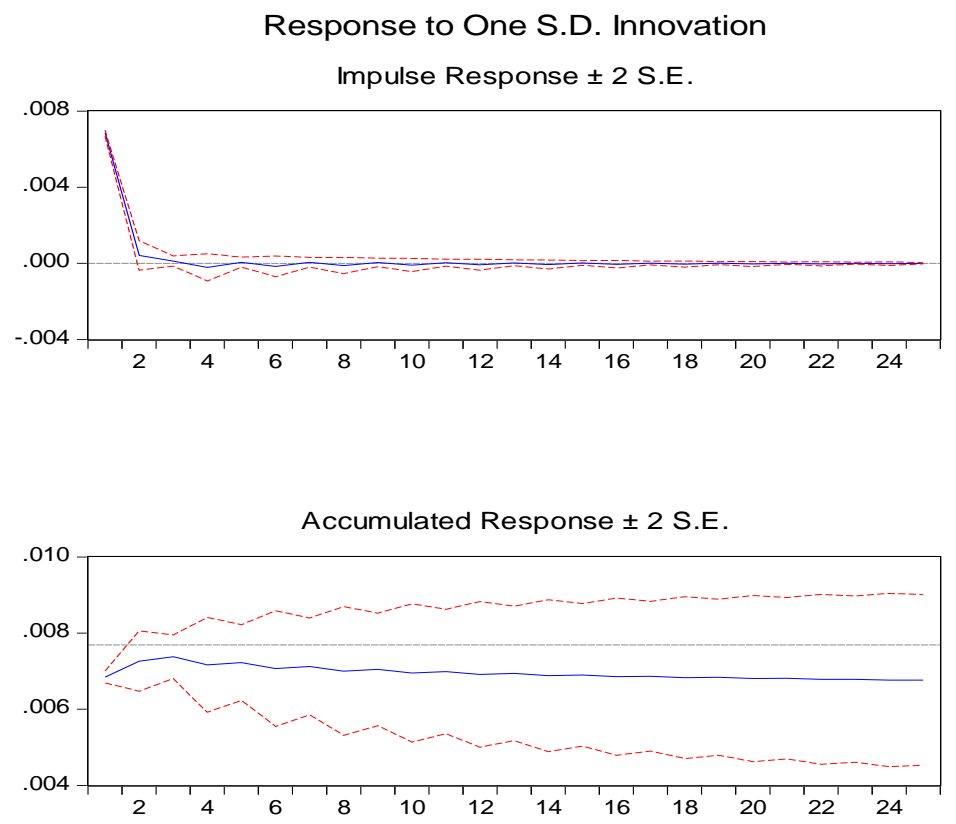

Figura 5. Prueba impulso respuesta del tipo de cambio en función de la tasa de interés Fuente: Elaboración propia en el software estadístico econometric-views.

Los resultados generados por los modelos ARFIMAX señalan la existencia de una relación entre el mecanismo de transmisión de la política monetaria (tasa de interés) y el canal de transmisión (volatilidad del tipo de cambio). Se encontró que la tasa de interés tiene un efecto transitorio sobre el premio al riesgo y que la volatilidad del tipo de cambio tiene un efecto permanente sobre el premio al riesgo, además la tasa de interés tiene un efecto permanente sobre la volatilidad del tipo de cambio. Estos eventos muestran que la política monetaria de Banco de México tiene diversas fases de propagación, primero a través de la tasa de interés que impacta directamente la volatilidad del tipo de cambio, y segundo, la rentabilidad del tipo de cambio que impacta inversamente al premio al riesgo de los activos financieros mexicanos.

\section{Conclusiones}

Se tiene evidencia de que los movimientos de la política monetaria a través de su mecanismo de transmisión, que es la tasa de interés, generan un efecto en la rentabilidad del tipo de cambio, que se identifica a su vez como canal de transmisión de la política monetaria.

Es decir, se tiene en un primer momento las decisiones de las autoridades monetarias implementadas mediante la fijación del valor de la tasa de interés, las cuales se transmiten de forma directa a través de la volatilidad del mercado cambiario. En un segundo momento se tiene que el comportamiento de la volatilidad del tipo de cambio genera una alteración que además es inversamente proporcional al valor del premio al riesgo de los activos financieros mexicanos, esto sugiere que la volatilidad del mercado cambiario realiza su función como canal de transmisión de la política monetaria en México.

Sin embargo, estimando un modelo conjunto para el caso del premio al riesgo en función de la tasa de interés y el tipo de cambio, la tasa de interés pierde poder explicativo, mientras que el tipo 
de cambio continúa fungiendo como el principal emisor de las consecuencias de la toma de decisiones del banco central dentro del premio al riesgo de los activos financieros en México.

Es así como la hipótesis de la existencia de una relación de largo plazo entre el mecanismo de transmisión de la política monetaria (tasa de interés) y el canal de transmisión (volatilidad del tipo de cambio) no se rechaza. Tampoco se rechaza que las políticas de la banca central afectan el mercado de capitales en este caso medido por el premio al riesgo, pues se encontró que la tasa de interés tiene un efecto transitorio sobre el premio al riesgo, efecto que se considera transmitido por el canal de transmisión que es la volatilidad del tipo de cambio el cual presenta un efecto permanente sobre el premio al riesgo. Estos eventos muestran que la política monetaria del Banco de México tiene esta importante fase de propagación y es por estás relaciones consideradas de largo plazo presentes entre las variables de estudio que se reconoce la existencia de la paradoja del banco central en la economía mexicana.

\section{Referencias}

[1] Alchian, A. A. (1950). Uncertainty, Evolution, and Economic Theory. Journal of Political Economy, 211221. DOI: https://doi.org/10.1086/256940

[2] Adenstedt, R. K, y Eisenberg, B. (1974). Estimación lineal de los coeficientes de regresión. Trimestral de Matemáticas Aplicadas, 32 (3), 317-327.

[3] Baillie, R. T. (1996). Procesos de larga memoria e integración fraccional en econometría. Revista de econometría, 73 (1), 5-59.

[4] Banxico. (2018). Educa Banxico. Obtenido de $\mathrm{ABC}$ de la política monetaria: http://educa.banxico.org.mx/pdfs/retobanxico/\%7B461B2467-EDA4-560B-46633568624E4F64\%7D.pdf

[5] Bekaert, G., Hoerova, M., y Lo Duca, M. (2013). Risk, uncertainty and monetary policy. Journal of Monetary Economics. DOI: https://doi.org/10.1016/j.jmoneco.2013.06.003

[6] Bernanke, B. S., \& Gertler, M. (2001). Should Central Banks Respond to Movements in Asset Prices? . The American Economic Review, 253-257. DOI: https://doi.org/10.1257/aer.91.2.253

[7] Blanco, G. P. (2006). La Memoria Larga y la Cointegración Fraccional en las Series Temporales; una Aplicación a la PAridad de Poder de Compra en los Países del Cono Sur. 1-49. DOI: https://doi.org/10.32468/dtseru.31

[8] Blinder, A. S. (1998). El banco central: Teoría práctica. Barcelona: Antoni Bosch.

[9] Bordo, M. D., \& Jeanne, O. (2002a). Boom-Busts in asset prices, economic instability, and monetary policy. National Bureau of Economic Research. DOI: https://doi.org/10.3386/w8966

[10] Bordo, M. D., y Jeanne, O. (2002b). Monetary Policy and Asset Prices: Does "Benign Neglect" Make Sense? . International Finance, 139-164. DOI: https://doi.org/10.1111/1468-2362.00092

[11] Borio, C. (2011). Implementing a Macroprudential Framework: Blending Boldness and Realism. Capitalism and Society, 6(1). DOI: https://doi.org/10.2202/1932-0213.1083

[12] Bomfim, A. N. (2003). Pre-announcement effects, news effects, and volatility: Monetary policy and the stock market. Journal of Banking and Finance, 133-151. DOI: https://doi.org/10.1016/s03784266(01)00211-4

[13] Cassoni, A. (1994). Cointegración (n. ${ }^{\circ} 330.115$ CASc). Universidad de la República (Uruguay). Facultad de Ciencias Sociales. Departamento de Economía. DOI: https://doi.org/10.24241/rcai.2019.121.1.181 
[14] Domowitz, I., y Hakkio, C. S. (1984). Conditional Variance and the Risk Premium in the Foreign Exchange Market. Journal of International Economics. DOI: https://doi.org/10.1016/00221996(85)90018-2

[15] Engel, C. (1995). The forward discount anomaly and the risk premium: A survey of recent evidence. Empirical Finance. DOI: https://doi.org/10.3386/w5312

[16] Gil León, J. M., \& Toca Toca, J. S. (2020). Política monetaria no convencional en EE. UU y comportamiento de los mercados emergentes en América Latina. Tendencias, 21(1), 24-51. DOI: https://doi.org/10.22267/rtend.202101.126

[17] Granger, J. (1980). An introduction to long memory property of stock market returns and a new model. Journal of Time Series Analysis, 1, 15-39.

[18] Greenspan, A. (1997). The Federal Reserve's Semiannual Monetary Policy Report. Washington, DC: Federal Reserve Board.

[19] Hosking, J. R. M. (1981). Fractional differencing. Biometrika 68 165-176. Mathematical Reviews (MathSciNet): MR614953 Zenmtralblatt MATH, 464. DOI: https://doi.org/10.1093/biomet/68.1.165

[20] c. Forecasting realised volatility using ARFIMA and HAR models. Quantitative Finance, 19(10), 16271638. DOI: https://doi.org/10.1080/14697688.2019.1600713

[21] Minsky, H. (1977). The Financial Instability Hypothesis: An Interpretation of Keynes and an Alternative to 'standard' theory. Journal of Economics and Business, 59-70.

[22] Mishkin, F. S. (1995). Symposium on the Monetary Transmission Mechanism. Journal of Economic Perspectives, 3-10.

[23] Rajan, R. G. (2006). HAS FINANCIAL DEVELOPMENT MADE DE WORLD RISKIER? European Financial Management, 499-533. DOI: https://doi.org/10.1111/j.1468-036x.2006.00330.x

[24] Roubini, N. (2006). Why Central Banks Should Burst Bubbles. International Finance, 87-107. D0I: https://doi.org/10.1111/j.1468-2362.2006.00032.x

[25] Salazar-Núñez, H. F. y F. Venegas-Martínez (2015). Memoria larga en el tipo de cambio nominal: evidencia internacional (1971-2012). Revista Contaduría y Administración, Vol. 60, No. 3, pp. 615-630. DOI: https://doi.org/10.1016/j.cya.2015.05.007

[26] Salgado, R. J., Herrera, F. L., y Ramírez, A. F. (2017). Cointegración fraccionaria entre el tipo de cambio, la Bolsa Mexicana de Valores y la TIEE. In A. R. Porras, y F. V. Martínez, Avances en economía financiera y desarrollo económico, Modelos analíticos y estudios cuantitativos. (pp. 87-114). México: Universidad de Guadalajara. DOI: https://doi.org/10.21919/remef.v12i1.17

[27] Samuelson, R. (2013, Octubre 28). La paradoja de Alan Greenspan al frente de la FED. Ciudad Autónoma de Buenos Aires, Argentina. DOI: https://doi.org/10.25085/rsea.780402

[28] Sriboonchitta, S., Sriboonjit, J., Chokethaworn, K., Chaitip, P., y Chaiboonsri, C. (2011). Forecasting of International Tourists' Expenditure in Thailand: Using AFIMAX and ARFIMAX-GARCH Approac. Thailand Econometrics Society, 220-232.

[29] Téllez-León, I. E. y F. Venegas-Martínez (2013). Modelado de las decisiones de política monetaria en un marco discreto, 2004-2012. Investigación Económica, Vol. 72, No. 284, pp. 23-55. DOI: https://doi.org/10.1016/s0185-1667(13)72591-8

[30] Titus, C. M., Wanjoya, A., \& Mageto, T. (2021). Time Series Modeling of Guinea Fowls Production in Kenya Using the ARIMA and ARFIMA Models. International Journal of Data Science and Analysis, 7(1), 1.

[31] Vasicek, O. A., and Venegas-Martínez, F. (2021). Modelos de la estructura de plazos de las tasas de interés: Revisión, tendencias y perspectivas. Revista Mexicana de Economía y Finanzas, Vol. 16, No. 2, pp. 1-15. DOI: https://doi.org/10.21919/remef.v16i2.587 